\title{
Coronary computed tomography angiography in children
}

\author{
LaDonna J. Malone ${ }^{1,2}$ (D) Cara E. Morin ${ }^{3} \cdot$ Lorna P. Browne $e^{1,2}$ \\ Received: 3 May 2021 / Revised: 16 August 2021 / Accepted: 10 September 2021 / Published online: 4 November 2021 \\ (c) The Author(s), under exclusive licence to Springer-Verlag GmbH Germany, part of Springer Nature 2021
}

\begin{abstract}
Imaging the coronary arteries of children, with their faster heart rates, small vessel size and common inability to lie still or breath-hold, has been a major challenge. With numerous advances in technology, CT examinations can now be performed quickly, often with children free-breathing and with much lower radiation doses than previously. This has led to increased use in children. Care must be taken with technique and choice of electrocardiogram (ECG)-gating technique to obtain adequate imaging for a diagnosis while keeping radiation dose as low as reasonably achievable (ALARA). In this paper, we discuss techniques and tips for CT imaging of the coronary arteries in children, including use of dual-source- and ultrawide-detector CT scanners.
\end{abstract}

Keywords Anomaly $\cdot$ Children $\cdot$ Computed tomography $\cdot$ Congenital heart disease $\cdot$ Coronary arteries $\cdot$ Dual-source computed tomography $\cdot$ Heart

\section{Introduction}

Computed tomography (CT) angiography is often the firstline diagnostic modality of choice for noninvasive imaging of the coronary arteries in both adults and children. CT is rapidly acquired and can be performed in the setting of multiple support devices. Compared with cardiac MRI and transthoracic echocardiography, CT provides excellent spatial resolution with superior visualization of the entire course of the coronary arteries [1].

Recent advances in CT technology have allowed for dramatic decreases in radiation dose while maintaining the excellent spatial resolution that allows for detailed anatomical evaluation. Acquisition of complete cardiac imaging in children typically requires less than $1 \mathrm{~s}$, obviating the need for sedation or breath-holding in many circumstances. The newest scanners allow for the modulation of multiple

LaDonna J. Malone

ladonna.malone@ childrenscolorado.org

1 Department of Radiology, University of Colorado, Aurora, CO, USA

2 Department of Radiology, Children's Hospital of Colorado, 13123 E. 16th Ave., B125, Aurora, CO 80045, USA

3 Department of Diagnostic Imaging, St. Jude Children's Research Hospital, Memphis, TN, USA components, including tube potential, tube current, pitch and gating, while new technologies include dual-source CT, ultrawide detectors and iterative reconstruction [2-7]. Manipulation of these components allows for reduced-dose radiation protocols in pediatric cardiac $\mathrm{CT}$, allowing for effective doses in the sub-millisievert $(<1 \mathrm{mSv})$ range.

Imaging of the coronary arteries in children with congenital and acquired coronary abnormalities is important for prognosis and treatment/surgical planning. This article describes the utility of CT for evaluating congenital anomalies of coronary origin and course, coronary abnormalities in Kawasaki disease, and pre- and postoperative evaluation in several scenarios.

\section{Technique}

\section{Scanners}

Dual-source CT scanners have two tubes at a $90^{\circ}$ angle, with second- and third-generation dual-source CT scanners achieving a temporal resolution of $66-75 \mathrm{~ms}$, which allows for imaging of the entire heart during a single heartbeat [8]. This improved temporal resolution has opened the possibility for imaging coronary arteries in pediatric patients with fast heart rates while keeping in line with the ALARA (as low as reasonably achievable) principle. 
Ultrawide-detector scanners have 256-320 row detectors and up to $160 \mathrm{~mm}$ in length of $\mathrm{z}$-axis coverage that enable scanning of the entire heart in a single volume acquisition. The temporal resolution has improved from 175 to $137 \mathrm{~ms}$ with newer scanners [9]. In the pediatric population, 80-mm $\mathrm{z}$-axis coverage has been shown to be effective for coronary artery imaging [10], 160-mm z-axis coverage in other studies $[11,12]$. Advantages of this technique include usually complete pediatric cardiac coverage without the need for table movement, resulting in a zero pitch (lower radiation), less motion artifact and no stairstep artifact. Radiation reduction in z-axis lengths less than the total detector width of $160 \mathrm{~mm}$ is a result of the ability to scan in axial mode, so there are less pitch-related overscanning, overlap and overbeaming compared to helical methods [13, 14]. These benefits are negated if the scan length is greater than $160 \mathrm{~mm}$ in the $\mathrm{z}$-axis because this exceeds the volume that can be scanned in the axial mode and requires helical scanning.

In summary, both types of modern scanners can be used to adequately evaluate coronary arteries in pediatrics with lower radiation doses compared to older-generation CT scanners. Meticulous attention to technique is needed to achieve optimum image quality and dose reduction. Dual-source CT has better temporal resolution, at $66-75 \mathrm{~ms}$ vs. $140 \mathrm{~ms}$ for volume scanners. However, volume scanners have larger $\mathrm{z}$-axis coverage without table movement, at $160 \mathrm{~mm}$ vs. 38.4-57.6 mm for dual-source CT [8].

\section{Patient preparation}

\section{Goal of imaging}

In pediatrics, if coronary artery imaging is requested, often the goal is identifying the origins and proximal course for congenital coronary anomalies for preoperative planning or to evaluate aneurysms or significant stenoses in postoperative patients or those with inflammatory disorders such as Kawasaki [8]. With modern scanners, this information can frequently be obtained while children are free-breathing [8]. However, if distal coronary artery course and caliber evaluation are desired by the clinical service, breath-holding often becomes necessary, requiring the use of general anesthesia in younger children.

\section{Sedation or general anesthesia}

With older-generation CT scanners, evaluation of the smallcaliber coronary arteries in young children often used to require sedation or anesthesia to compensate for respiratory motion and fast heart rates. The improved temporal resolution of modern CT scanners now allows for free-breathing acquisitions for most indications. Children ages 6-7 years and older can generally comply with breath-holding instructions and do not require sedation. For very young infants ( $<1$ month), no sedation is typically needed when using the feed-and-swaddle method or giving sucrose on a pacifier during the exam. For older infants and children younger than 6-7 years, imaging is often obtained while children are sedated but free-breathing [3, 10, 11, 15-24]. The use of general anesthesia with suspended respiration is occasionally required for children younger than 6-7 years, especially for evaluating the distal coronary arteries [25].

\section{Heart rate and heart rate variability}

Pediatric patients typically have much faster heart rates than adults, and they often respond variably or inadequately to medications used to decrease heart rates such as beta-blockers. Studies have found that in heart rates below $70-75$ beats per minute (bpm), optimum imaging with limited cardiac motion is obtained in end-diastole, at $70 \%$ of the RR inter$\mathrm{val}$, whereas with faster heart rates imaging is geared toward end-systole, at 30-40\% of the RR interval [16, 19, 23].

Heart rate variability can also cause some difficulty with the timing of exams, particularly methods such as prospective-gated step-and-shoot methods. Less heart rate variability and typically lower heart rates occur in children under general anesthesia $[5,26]$. Some institutions consider $<6-10 \mathrm{bpm}$ variation to be low variability $[5,12]$. One study did not see a difference in the visualization of coronary arteries with increased variability [26].

\section{Medication for fast heart rates}

In many adult studies, medications were given to decrease heart rate, most commonly beta-blockers [27] and vasodilators such as nitroglycerin [28], or both [9, 29]. This is not as frequently done in the pediatric population. The use of betablockers has been reported for imaging coronary arteries in pediatrics by Han et al. [5] for a goal heart rate of $60 \mathrm{bpm}$ and by Le Roy et al. [12] for a heart rate of $>70 \mathrm{bpm}$. In a case report of a child with Kawasaki disease without adequate response to beta-blockers for reducing heart rate and developing hypotension, the authors described the use of phenylephrine to achieve the desired heart rate for imaging [30].

\section{Additional technical considerations}

As we continue to push for lower radiation doses for CT scans, meticulous technique is required for optimal image quality and for dose-reducing modulation techniques on the scanner to work correctly. For instance, streak artifacts from contrast agent flowing into the heart are increased at lower kilovoltage peak, and the presence of contrast agent in the left innominate vein can affect image quality. This can be 
decreased with different techniques, including preference for a lower-extremity intravenous (IV) line, contrast/saline mixture to decrease the density of contrast agent during infusion (see contrast section), or scan direction in $\mathrm{z}$-axis beginning opposite of the injection, i.e. caudocranial for an upper-extremity IV line and craniocaudal for a lowerextremity IV line [18]. An additional factor is beam-hardening artifact, which increases at a lower kilovoltage peak. This artifact be reduced by having the child's hands overhead instead of beside the chest for the scan. Additional artifacts from electrocardiogram (ECG) leads and other support and monitoring devices can affect image quality, particularly at lower kilovoltage peaks ( $70-80 \mathrm{kVp}$ ). ECG leads should be placed away from the thorax, on the arms and abdomen, to reduce this $[8,16]$. Because of the material differences in ECG leads, some types cause worse artifacts than others, and some leads that are safe for use in MRI also have fewer streak artifacts when used in CT. Also, the child must be in the isocenter of the scanner for optimal dose modulation with modern scanners [8].

\section{Electrocardiogram gating}

The type of ECG gating used is important in determining image quality for the exam and, along with z-axis coverage discussed later, is what determines radiation dose for these examinations.

\section{Retrospective gating}

Scanning has been performed using retrospective ECG gating with full dose throughout the cardiac cycle, which has the highest radiation dose, or with dose modulation. With dose modulation, higher radiation is used during a short period of the RR interval and a much lower dose is used for the remainder. The radiation dose for the scan is dependent upon the amount of dose administered throughout the exam. In pediatrics, dose-modulated retrospective ECG gating is typically used with some techniques, ranging from full dose at optimum stillness based on heart rate to $4 \%$ of the dose for the remainder of the cardiac cycle [19].

\section{Prospective gating}

With newer scanners, success rates in the visualization of coronary arteries in pediatrics have been shown using prospective techniques, including step-and-shoot and ECG-triggered exams. Step-and-shoot technique acquires images over three or more consecutive heartbeats and is susceptible to heart rate variability and motion artifact including stairstep artifact. Step-and-shoot technique has been associated with a significant rate of stairstep artifact because it obtains data
Table 1 Estimated effective dose for dual-source computed tomography (DSCT) comparison on non-electrocardiogram (ECG)-gated and high-pitch spiral ECG-triggered examinations

\begin{tabular}{lll}
\hline DSCT & Age (y) & $\begin{array}{l}\text { Effective } \\
\text { dose (mSv) }\end{array}$ \\
\hline Non-ECG-gated & & \\
Ben Saad et al. [15] & $<1 \mathrm{y}$ & 0.5 \\
Jin et al. [19] & $<5 \mathrm{y}$ & 0.74 \\
Kanie et al. [20] & $<6 \mathrm{y}$ & 1.3 \\
Liu et al. [21] & DSCT & 0.47 \\
High-pitch spiral ECG-triggered & & \\
Han et al. [5] & $<18 \mathrm{y}$ & 0.24 \\
Li et al. [31] & $<4 \mathrm{y}$ & 0.4 \\
Liu et al. [21] & $<5 \mathrm{y}$ & 0.41 \\
Malone et al. [26] & $<20 \mathrm{y}$ & 0.98 \\
\hline
\end{tabular}

$m S v$ millisieverts, $y$ years

over several cardiac cycles, during which time patient position can change and limit visualization of coronary arteries in some cases. In heart rates $<75 \mathrm{bpm}$, optimum visualization of coronary arteries is typically at end-diastole (at 70\% of the RR interval), while with faster heart rates, visualization is best at end-systole (at approximately $40 \%$ of the RR interval), which is most often the case in pediatric patients. The radiation dose is again dependent on the amount of cardiac cycle imaged as well as $\mathrm{z}$-axis coverage, with some institutions using a relatively narrow window $(30-40 \%$ of the cardiac cycle) and others a wider window (40-80\% of the cardiac cycle) or a form of dose-modulated scanning from $25 \%$ to $85 \%$ of the cardiac cycle, with a higher dose at optimum stillness based on heart rate and the remainder at $20 \%$ mAs $[12,26]$.

While there is overlap in techniques of ECG gating for retrospective and prospective gating with scanner types, there are notable differences in the rapid type of imaging that can be done with ECG gating for two scanner types: highpitch spiral mode for dual-source CT and target mode for ultrawide detectors, both using prospective ECG triggering.

\section{High-pitch spiral prospective gating}

The lowest radiation dose type of ECG-gated scan on dualsource CT is high-pitch spiral with prospective ECG triggering, which uses a pitch of 3.2-3.4 and is accomplished without artifact because of the dual-source detectors. The goal is to image at $40 \%$ of the RR interval for faster heart rates and at end-diastole (at 70\% of the RR interval) for lower heart rates. This technique can be done with a similar effective dose to non-ECG-gated studies, as shown in Table $1[5,15$, 19-21, 26, 31]. Adequate visualization of coronary arteries is often achieved at lower heart rates with little heart rate 
variation, with some centers using this technique if heart rates are $<60 \mathrm{bpm}$ and have $<10 \mathrm{bpm}$ variation [6].

\section{Target electrocardiogram-gated}

On ultrawide detector scanners, a type of prospective ECG-triggered scan obtains images at a point in the cardiac cycle without padding, which allows for further radiation dose reduction. This scan can image up to $160 \mathrm{~mm}$ without table motion, so there are no stairstep artifacts, and in less than one cardiac cycle, which reduces motion artifact [14]. Similar heart-rate-based targets are used. This technique involves the use of halfscan reconstruction of raw data as a post-processing technique to find a motion-free phase within the rotation time of $270-350 \mathrm{~ms}$.

\section{Non-electrocardiogram-gated}

Non-ECG-gated exams have also been reviewed for evaluation of coronary arteries in pediatrics, with variable results: Ben Saad et al. [15] visualized $43 \%$ of the left coronary artery and $15 \%$ of the right; Kanie et al. [20] visualized $80.5 \%$ of proximal segments of both coronary arteries.

\section{Z-axis coverage}

After deciding on the adequate type of ECG gating for coronary artery evaluation, one must select coverage in the $\mathrm{z}$-axis (head-to-foot direction) that is adequate for visualization. For instance, if coronary artery origins are the clinical question, the field of view (FOV) could be limited to several centimeters centered at the aortic root level, whereas if the origins and entire course of the coronary arteries is required, then coverage must include the bottom of the heart, and a typical coverage might be from the level of the aortic arch through the cardiac apex. On some occasions, there are multiple clinical questions for the scan, requiring a larger FOV in the z-axis. If retrospective or prospective gating with padding is going to be used, a second scan with a larger FOV, either without ECG gating or with high-pitch spiral or "target" mode, might be used for the larger FOV scan in order to limit radiation dose.

\section{Radiation dose and scan parameters}

The radiation dose for coronary $\mathrm{CT}$ scan varies greatly based upon the type of ECG gating used, with retrospective ECG gating having the highest radiation doses, and high-pitch spiral or target the lowest, and prospective gating methods in the middle. The length of $\mathrm{z}$-axis coverage plays a significant role in radiation dose, as mentioned. One paper with ranges of effective dose estimates for dual-source CT using different ECG-gating methods listed high-pitch spiral at $0.2-0.5 \mathrm{mSv}$, prospective ECG sequential (step-and-shoot) at $0.3-1 \mathrm{mSv}$, and retrospective ECG gated with dose modulation at $2-5 \mathrm{mSv}$ [8]. For second- and third-generation dual-source CT scanners, a comparison of non-ECG-gated and high-pitch spiral ECGtriggered scans shows a similar effective dose (Table 1). For wide-row volume - 256-512-slice - scanners, published effective dose for prospective ECG-triggered exams has ranged 0.97-1.65 $\mathrm{mSv}$ [10-12].

With modern scanners, there are weight-based protocols for getting adequate images for diagnostic purposes (including acceptable noise, etc.), with many scanners having weight ranges for kilovoltage peak $(\mathrm{kVp})$ selection as well as dose-modulated milliampere-seconds (mAs) parameters. Remember that the "k-edge" of iodine is at $70 \mathrm{kV}$. Additional considerations are needed for children with implanted materials (e.g., stents, pacemaker leads) that cause more streak artifacts at lower radiation levels, so sometimes a higher $\mathrm{kVp}$ is used, but the balance between adequate evaluation and radiation dose must always be maintained.

\section{Contrast injection}

\section{Intravenous catheter}

Although some studies report injecting into a central venous catheter, the majority of studies evaluating coronary arteries reported using a peripheral venous catheter, with the right antecubital vein being most common, employing a 24-gauge in infants and up to an 18-gauge in adolescents [8]. Size of peripheral catheter affects the injection rate. Central venous catheters and peripheral venous catheters have a different scan start time [3, 15]. Power injection is vital for uniform contrast injection rates and can be done safely with parameters set up for pressure thresholds to stop injecting if there is potential extravasation of contrast agent.

Low osmolar or iso-osmolar contrast agents are used with 300-370 mg iodine/mL, with typical doses ranging $1.5-2 \mathrm{~mL} / \mathrm{kg}$, for coronary artery imaging [8]. Injection rates vary from $0.5-5 \mathrm{~mL} / \mathrm{s}$ to rates of $0.5-2 \mathrm{~mL} / \mathrm{s}$ for younger children with smaller catheter sizes. A saline bolus immediately follows contrast injection, typically a volume of $10-20 \mathrm{~mL}$ using the same injection rate as the contrast agent; this is considered a biphasic injection and is used at many centers $[10,12,19,21,23]$. Other 
injection methods include triphasic injection, with the first phase being full-strength contrast agent, the second phase diluted contrast agent or full-strength contrast agent at a slower injection rate, and the third phase being a saline flush $[8,17]$. Some centers use a contrast dilution mixture for infants $<4 \mathrm{~kg}$ with a minimum dose of $7 \mathrm{~mL}$ contrast mixed with saline at a ratio of $7: 3$ followed by saline bolus [19].

\section{Scan timing}

For coronary artery imaging, we aim for first-pass contrast agent in the aorta. Scan timing can be done by placing a region of interest (ROI) in the left ventricle or within the ascending or descending aorta. The scan start time can be set based upon the length of contrast injection, automatically triggered with a threshold value set for the ROI (usually $100-150$ Hounsfield units [HU]) or manually by a technologist or radiologist at the scanner [8, 11, 12, 21, 23, 26, 31]. Some institutions have standard start times and adjust the rate of contrast injection accordingly, or set the start time based on the end of contrast injection [3, 8, 10, 15, 19, 20, 22]. The timing of scan start varies slightly with different scanners or protocols, depending on delay time, table movement, etc.

\section{Scan reconstruction}

Filtered back-projection has been a commonly used algorithm for providing simple, rapid imaging reconstruction, but newer iterative reconstruction methods have become more widely used in pediatrics [8, 12, 22, 23]. These include sinogram affirmed iterative reconstruction (SAFIRE) and adaptive statistical iterative reconstruction (ASIR). SAFIRE has been shown to have improved image quality and signal-to-noise ratio (SNR) as well as decreased noise when compared to filtered back-projection $[8,22]$.

\section{Post-processing}

Post-processing is vital for adequate evaluation of coronary arteries. This is often performed on a separate workstation or software. Three-dimensional (3-D) reconstructions can aid in clinical interpretation and are also appreciated by clinical colleagues and surgeons. Orthogonal planes, including the plane of the aortic root, are especially helpful for evaluating coronary artery origins. Additional aspects that can be useful in select cases are "curved plane reformat" multiplanar reconstruction, which lays out the coronary artery and a virtual fly-through of the intraluminal coronary artery from its origin.

\section{Common indications for imaging coronary arteries in the pediatric population}

\section{Anomalies of coronary artery origin and course}

The spectrum of coronary artery anomalies ranges from benign/incidental findings to significant cardiac morbidity in infancy or increased risk of cardiac sudden death in childhood or adolescence [32]. Abnormalities can be found in the coronary artery origin, course, and size or the number of vessels. Isolated anomalies of coronary artery origins, in those without structural heart disease, had an estimated incidence of $0.7 \%$ in the general population in a review of 77 studies [33]. Two of these are anomalous aortic origin of the right coronary artery from above the left sinus of Valsalva with interarterial course (between the aorta and pulmonary artery) and anomalous left coronary artery from above the right sinus of Valsalva. Incidence of anomalous right coronary artery from above the left sinus is relatively more common (incidence $0.23 \%$ ) than anomalous left coronary artery (incidence $0.03 \%$ ), although anomalous left coronary artery has a higher incidence of sudden cardiac death, estimated at $38-66 \%$ [33]. Coronary artery origins can often be seen on echocardiogram, although advanced cross-sectional imaging is often obtained for further evaluation or when the coronary artery origins are not seen on echo in the setting of chest pain [1,34]. Once an anomalous coronary artery origin is seen, determining the course is important for management decisions. Interarterial course (between aorta and pulmonary artery) has higher association with sudden death than retroaortic or prepulmonic courses [34]. Also, an intramural course of the coronary artery has important implications for potential therapy. Although the direct findings of coronary artery coursing in the aortic wall are not easily determined by $\mathrm{CT}$, indirect findings that correlate with intramural course include acute angulation of origin with slit-like ostium and narrowed oblong shape of the coronary artery [34].

Treatment decisions are complex and can be medical or surgical. Surgical treatment options include unroofing in the setting of intramural course (Fig. 1), translocation/reimplantation, or coronary artery bypass graft $[34,35]$. Many anomalous left coronary arteries with intramural course can be treated with surgical unroofing, and while anomalous right coronary artery treatment is the same, the procedure is more controversial given the lower incidence of sudden cardiac death. In a single-institution prospective cohort of 44 patients who underwent surgery including both anomalous left and anomalous right coronary arteries, one required reintervention and 91\% (40/44) were asymptomatic after surgery, with 42 returning to full activity [35].

Coronary artery anomalies have a higher incidence in children with congenital heart disease, ranging from an 

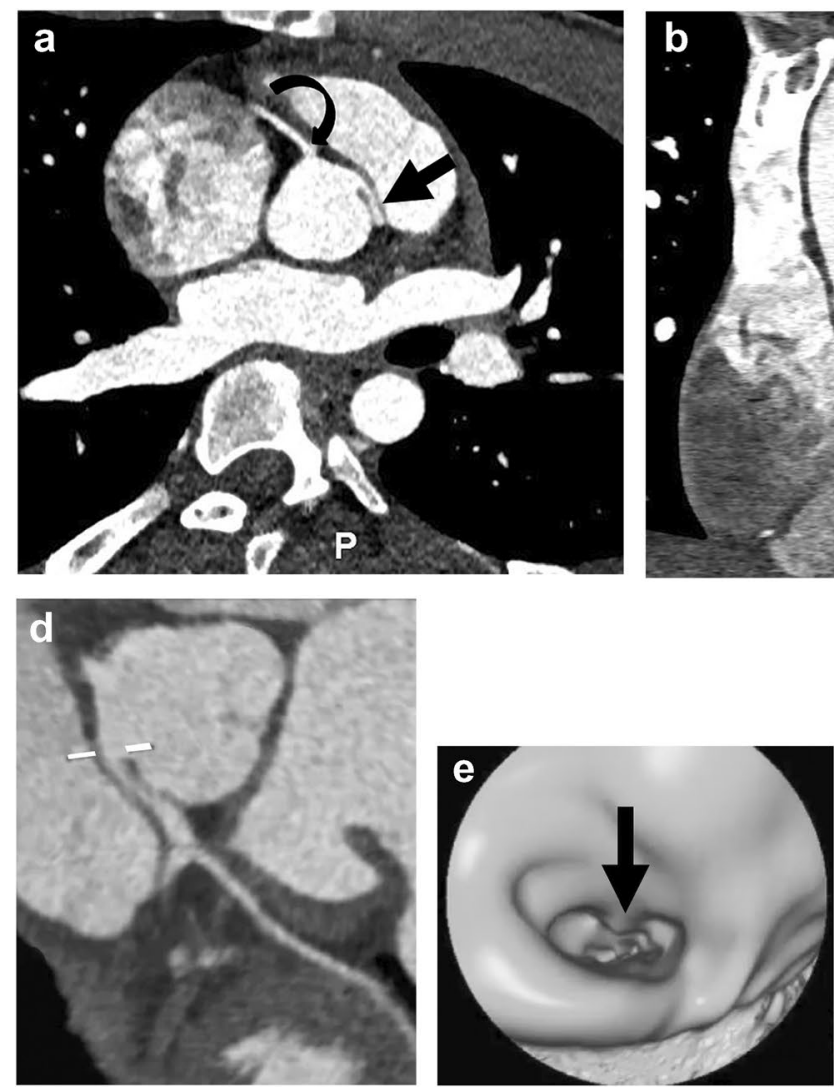

Fig. 1 Anomalous origin of the left coronary artery in a 16-year-old boy with syncope. a Oblique reconstruction from CT angiography in aortic root plane shows anomalous origin of the left coronary artery from above the right sinus of Valsalva with interarterial course and likely intramural course (straight arrow). Right coronary artery origin is normal (curved arrow). $P$ posterior. b Coronal oblique reconstruction from CT angiography demonstrates oblong shape of the left coronary artery (arrowhead), taller than wide, suggesting intramural course. c Three-dimensional (3-D) reconstruction image from CT angiography viewed from the anterior demonstrates coronary artery origins and proximal course, with the anomalous left coronary artery originating above the right sinus (arrow). The pulmonary artery and ascending aorta were removed for adequate coronary artery visualiza-

incidence of 5\% in those with coarctation [36] to $7 \%$ in tetralogy of Fallot [35] and up to $37 \%$ in single-ventricle physiologies [36]. Identifying these anomalies has become an important part of the preoperative evaluation to avoid inadvertent injury and has been shown to be effectively diagnosed with modern CT techniques compared to surgical visualization and catheter angiography $[36,37]$. An example of preoperative CT evaluation of coronary anatomy in an infant with a diagnosis of congenital heart disease is shown in Fig. 2. Coronary artery anomalies in children with congenital heart disease can alter the surgical approach and even the surgical options, and preoperative imaging can aid in surgical planning and counseling prior to surgery $[36,37]$.
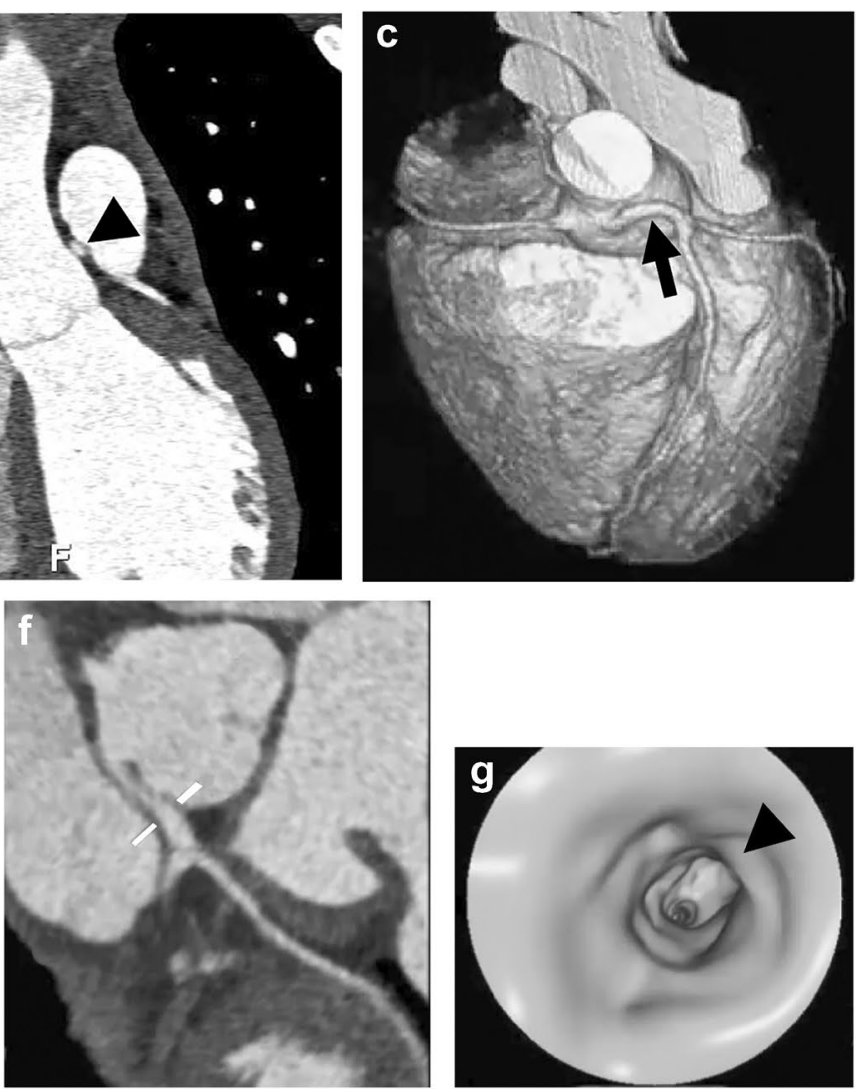

tion. d Curved planar reformat from CT angiography shows horizontal lines indicating level for virtual fly-through image. e Intraluminal virtual fly-through image from CT angiogram viewed from inside the aorta looking right at the level shown in (d). The intraluminal image of the proximal left coronary artery demonstrates oblong shape (arrow) of the coronary artery proximally. f Curved planar reformatted image from CT angiography shows horizontal lines at the level of the virtual fly-though image further distally in the coronary artery. $\mathbf{g}$ Intraluminal virtual fly-through image from CT angiography viewed from inside the right coronary artery at the level shown in (f) in the more distal left coronary artery, where it regains its rounded shape (arrowhead)

More rare coronary artery origin anomalies include anomalous left coronary artery from the pulmonary artery (ALCAPA) and, even more rare, anomalous right coronary artery from the pulmonary artery (ARCAPA). Children with ALCAPA are usually symptomatic in the first year after birth because of coronary steal [38]. Less than half of people with ARCAPA in adulthood were found to be symptomatic (41\%) and $40 \%$ had ischemia [38]. Figure 3 shows images of an infant with ALCAPA.

\section{Kawasaki disease}

Kawasaki disease is a febrile vasculitis of unknown origin that occurs primarily in children younger than 5 years and 


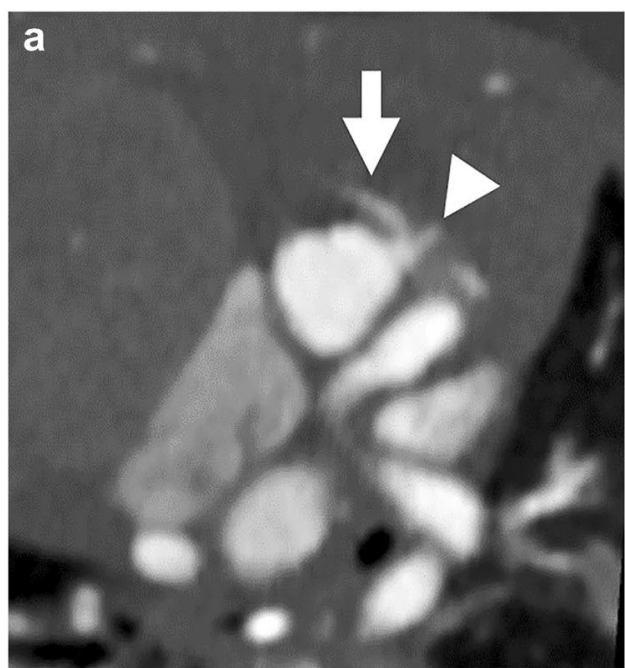

Fig. 2 Tetralogy-of-Fallot-like double-outlet right ventricle with probable single coronary artery in a 1.5-month-old girl. a Axial oblique reconstruction from CT angiography in plane of aortic root shows a coronary artery originating from the left sinus with trifurcation: right coronary artery (arrow); left anterior descending artery (LAD; arrowhead); and prominent diagonal branch. b The diago-

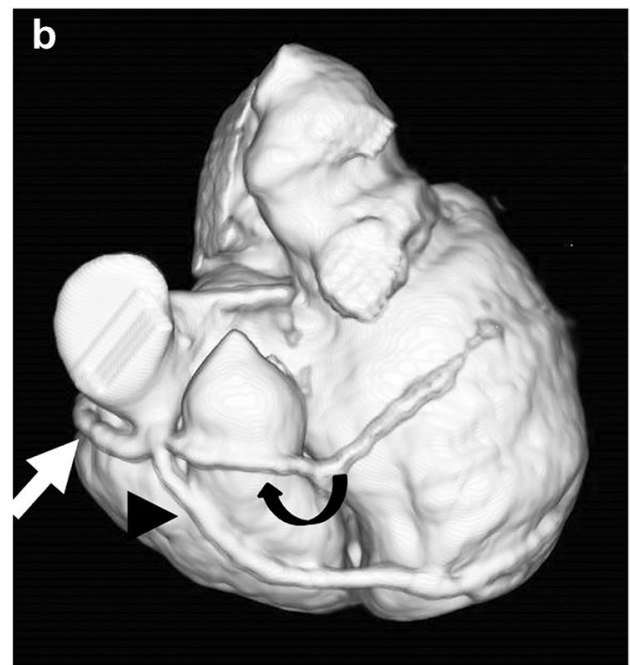

nal branch is better visualized on three-dimensional reconstruction. Three-dimensional reconstruction from CT angiography viewed from the anterior left shows coronary artery origin and trifurcation with right coronary artery (straight arrow), LAD (arrowhead) and prominent diagonal branch (curved arrow). The circumflex artery originated separately (not shown)
Fig. 3 Anomalous left coronary artery from pulmonary artery (ALCAPA) in a 3-month-old girl with cardiac dysfunction and concern for coronary anomaly. a Axial oblique CT angiogram shows anomalous left coronary artery (arrowhead) originating from the pulmonary artery (asterisk). b Coronal oblique CT angiogram shows left coronary artery (arrow) originating from the pulmonary artery (asterisk), known as ALCAPA
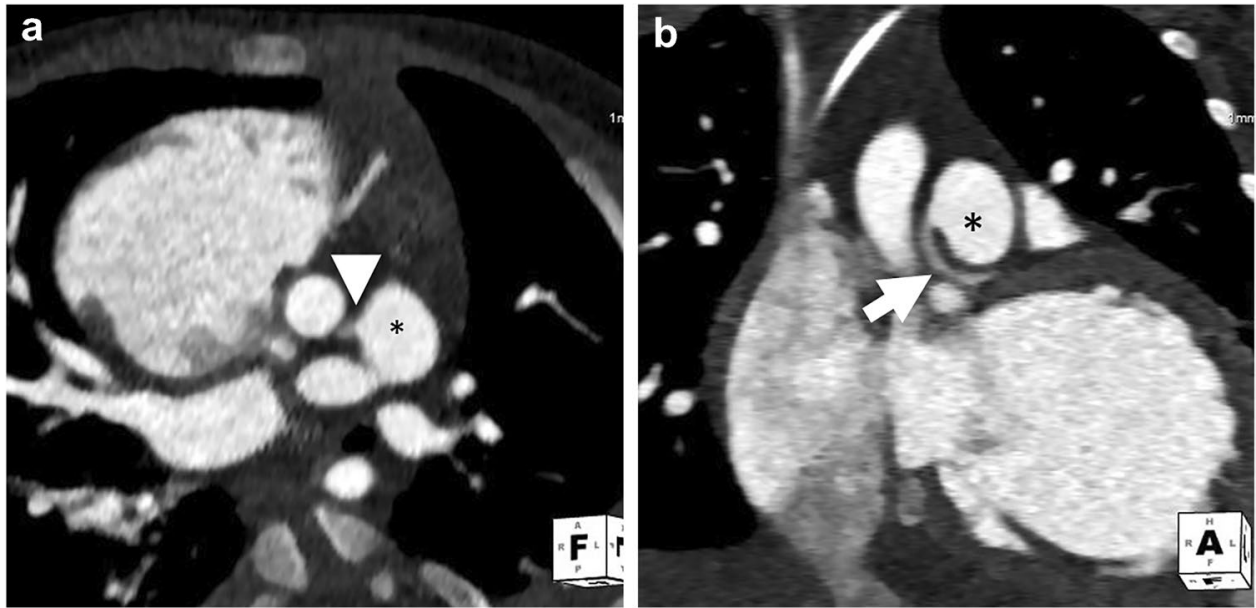

is now the most common cause of acquired heart disease in children in the United States [39]. During the acute illness, some children experience necrotizing arteritis, predominantly of the coronary arteries. A combination of destruction of the arterial walls, ongoing inflammation, and development of thrombus leads to acute and chronic cardiovascular complications [39]. As a result, up to $25 \%$ of these children develop coronary artery aneurysms, which can thrombose or develop significant stenosis [8, 39]. With treatment, the prevalence of coronary artery abnormalities can be reduced to 4-8\% [40, 41]. Prognosis and management of these children require detection and quantification of coronary artery aneurysms and stenoses, typically with echocardiography, which has high sensitivity and specificity for detecting abnormalities in the proximal coronary artery segments [39]. However, the utility of echocardiography is more limited for detecting distal coronary artery stenoses, thrombi and distal coronary involvement [39]. CT is widely used to obtain high-resolution imaging of the entire coronary anatomy [8]. Figure 4 demonstrates multiple coronary artery aneurysms in the right coronary artery in a child with Kawasaki disease as well as progression with development of stenoses on follow-up. 
Fig. 4 Kawasaki disease and right coronary artery aneurysms in an 8-year-old boy. a Curved planar reformat from $\mathrm{CT}$ angiography of the right coronary artery $(R C A)$ demonstrates a smaller aneurysm proximally (arrow) and a large aneurysm with coarse calcifications distally (arrowhead). b Three-dimensional reconstruction from CT angiography viewed from the right anterior also demonstrates a proximal aneurysm (arrow) and distal aneurysm with calcifications (arrowhead). c Curved planar reformat from CT angiography on follow-up 6 years later at 14 years old demonstrates increased calcification peripherally in both aneurysms as well as new high-grade stenosis just proximal to the proximal aneurysm (arrow) and a nearly non-opacified coronary artery segment between the aneurysms (arrowhead). d Coronal oblique reconstruction from $\mathrm{CT}$ angiography demonstrates high-grade stenosis (arrowhead) and near occlusion of the artery between the aneurysms (arrow)
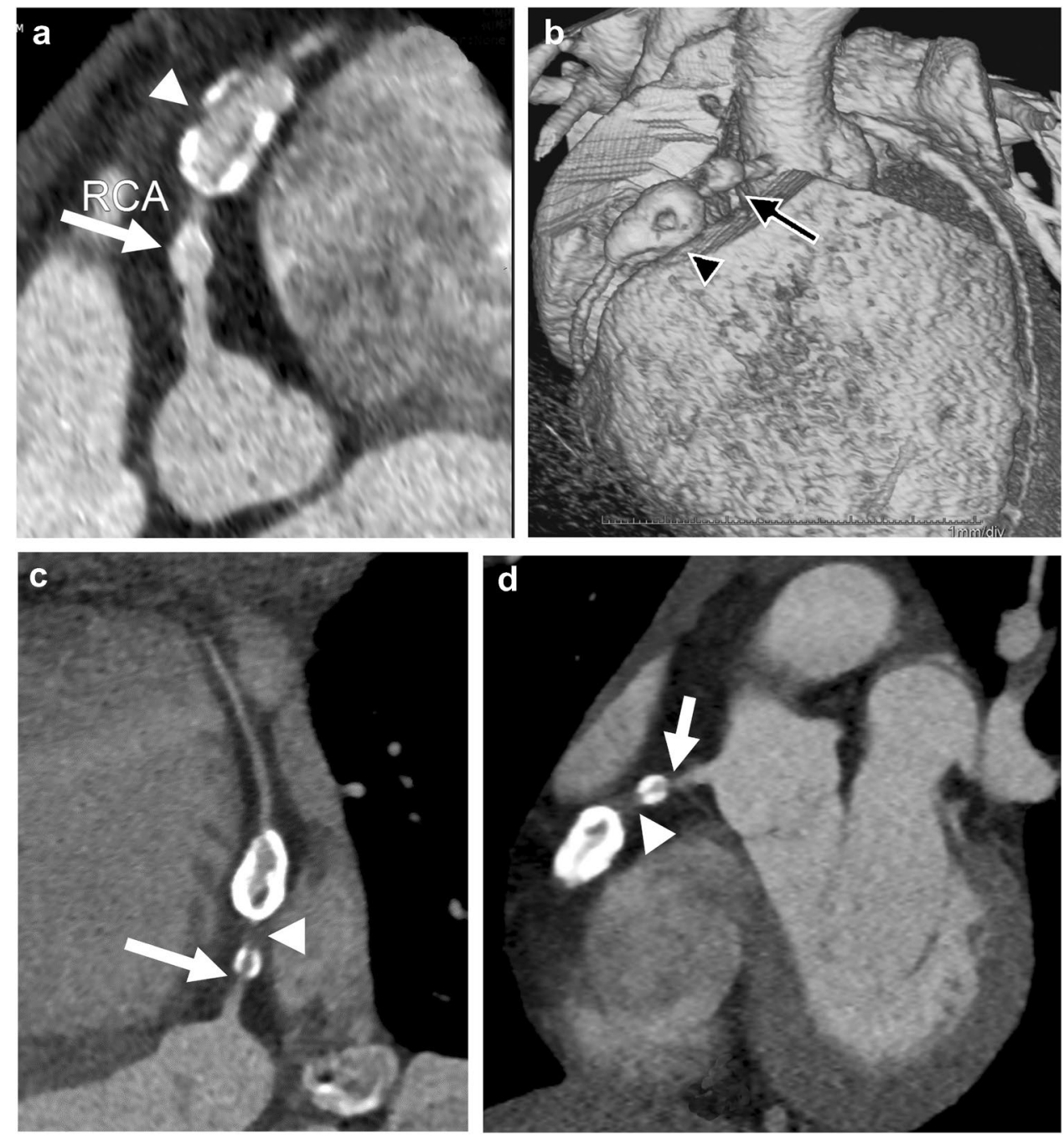

Children who experience the multisystem inflammatory syndrome in children (MIS-C) associated with the severe acute respiratory syndrome coronavirus 2 (SARS-CoV-2) disease of 2019, or COVID-19, have similar coronary artery manifestations to children with Kawasaki disease. Approximately $6-24 \%$ of children with MIS-C have coronary artery dilation or aneurysms [42].

\section{Postoperative patients}

Several complex congenital heart surgeries require coronary artery manipulation and sometimes relocation. These include the arterial switch procedure with Lecompte maneuver for transposition of the great arteries and the Ross or Ross Konno procedure for aortic valve stenosis. Both procedures involve excision of the coronary arteries with re-implantation, which can be associated with both early and late complications related to impaired coronary flow. Coronary artery evaluation by $\mathrm{CT}$ is the noninvasive method of choice for evaluating these children if there is clinical suspicion for complication. All people who have undergone coronary artery manipulation should be evaluated for coronary artery patency and anatomical integrity at least once in adulthood, and CT angiography is often the noninvasive method of choice for this evaluation [43]. Particular attention should be paid to origins and proximal course. Examples of a case in the acute postoperative setting are shown in Fig. 5 and in the late postoperative period, years after surgery, in Fig. 6.

Computed tomography angiography is also useful in the postoperative evaluation of coronary artery bypass grafts and stents $\geq 3 \mathrm{~mm} \mathrm{[44]} \mathrm{and} \mathrm{to} \mathrm{assess} \mathrm{for} \mathrm{coronary}$ allograft vasculopathy in children post heart transplant [45]. Figure 7 shows a child with two prior heart transplants and findings thought to represent coronary artery vasculopathy. 

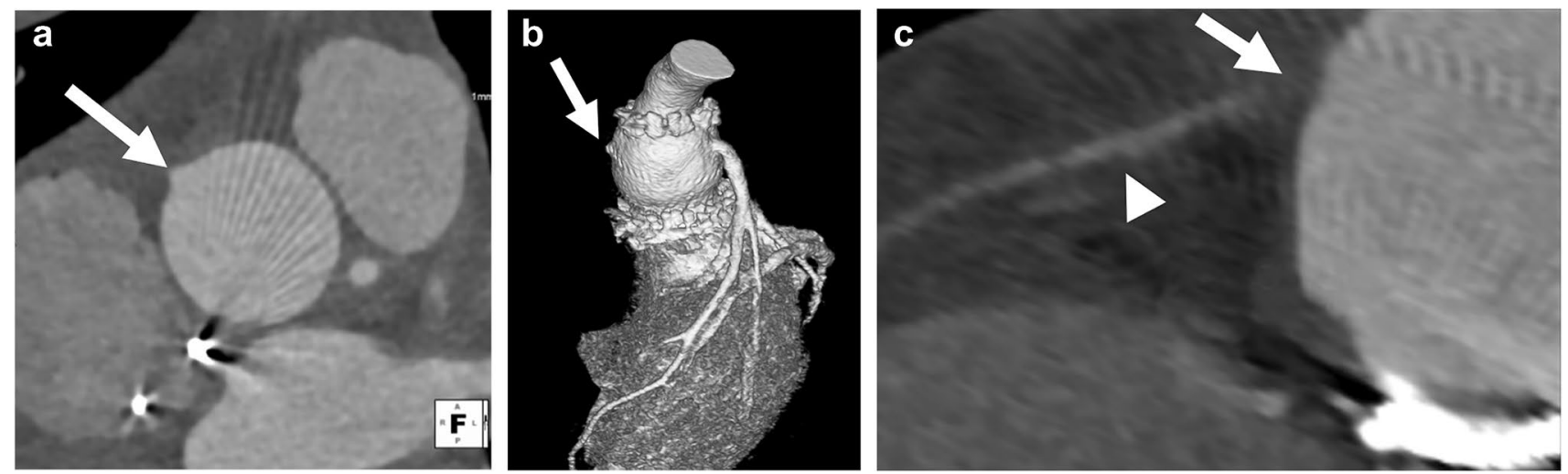

Fig. 5 Early postoperative imaging in a 10-year-old boy with LoeysDietz syndrome status post aortic root replacement. Echocardiography in the acute postoperative period showed a flared tricuspid valve with right coronary artery injury by retraction in the operating room and subsequent repair. a, b Orthogonal reconstruction of CT angiography in the plane of the aortic root (a) and three-dimensional
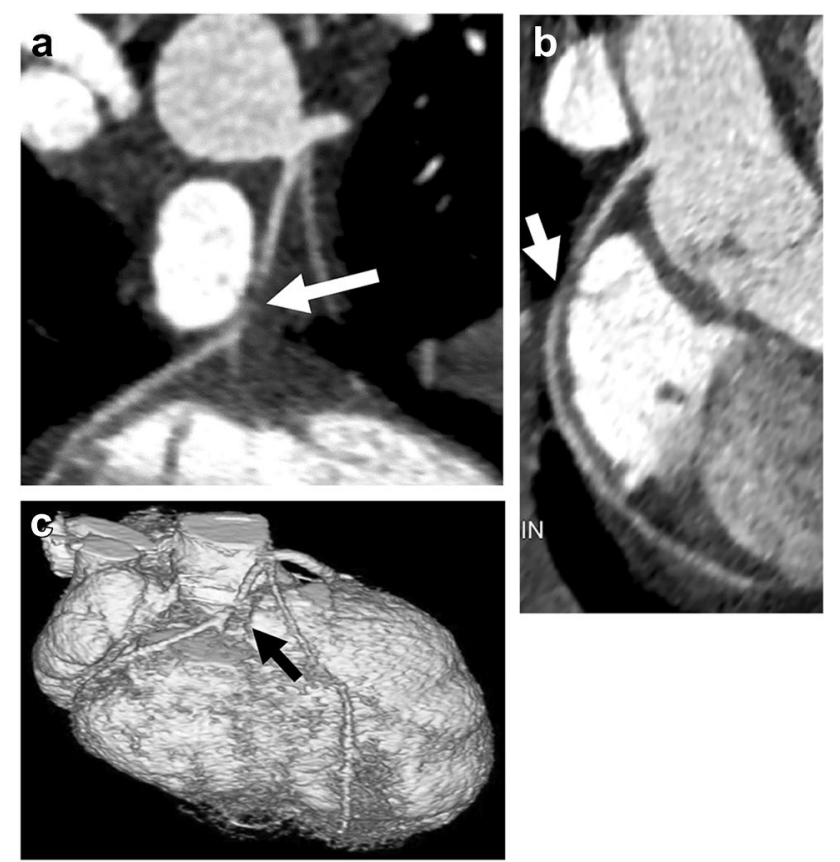

Fig. 6 Late postoperative imaging in an 8-year-old girl with a history of D-transposition of the great arteries and coarctation status post repair, imaged for routine evaluation. a, b Coronal (a) and sagittal oblique (b) reconstructions from CT angiography demonstrate decreased caliber of right coronary artery as it courses around the main pulmonary artery to the right atrioventricular groove (arrows). c Three-dimensional reconstruction from CT angiography, viewed from the anterior with the pulmonary artery and ascending aorta removed above the sinotubular junction, also demonstrates right coronary artery narrowing (arrow). This focal narrowing corresponded to an area of decreased myocardial perfusion in the right coronary artery distribution on subsequent stress cardiac MR (not shown). This was thought to be a result of mass effect from the pulmonary artery reconstruction of CT angiogram viewed from the left (b) demonstrate abrupt cutoff of the right coronary artery at its origin (arrows). c Multiplanar reconstruction of the right coronary artery from CT angiography shows no opacification of proximal right coronary artery (arrow), with reconstitution of the vessel distally (arrowhead)

\section{Coronary artery compression from epicardial leads}

Epicardial leads placed in pediatric patients can result in complications related to adhesion of the leads to the epicardial surface as the child grows, causing cardiac strangulation or coronary artery compression and risk of sudden death [46]. What was thought to be a rare complication of epicardial leads in case reports was shown by Mah et al. [47], in a retrospective review of 145 patients, to have an incidence of 5.5\%. Chest radiography has been proposed as an initial screening tool, with a classic pattern of cardiac strangulation defined as a heart-shaped orientation of the atrial and ventricular leads within the cardiac silhouette on an anteroposterior/posteroanterior radiograph [46, 47]. Asymptomatic pediatric patients with epicardial leads are screened with periodic chest radiographs. Children with suspicious findings on chest radiography or those with clinical suspicion for coronary artery compression can undergo noninvasive imaging with CT angiography [47] (Fig. 8).

\section{Conclusion}

The use of coronary CT angiography in children has grown with technological advances, including faster scans and better ECG gating. To achieve high-quality images in children, radiologists must optimize techniques for the child, CT scanner and clinical indication. We have reviewed methods for technique optimization and the common indications for imaging coronary arteries in children. 
Fig. 7 Coronary artery vasculopathy in a 17-year-old girl status post heart transplant. a Sagittal maximum-intensity projection CT angiogram shows significant tortuosity of the left anterior descending (LAD) artery (arrow) as well as tortuous branches thought to be secondary to coronary artery vasculopathy. b Three-dimensional reconstruction from $\mathrm{CT}$ angiography viewed from above shows tortuous mid and distal LAD artery (arrow)
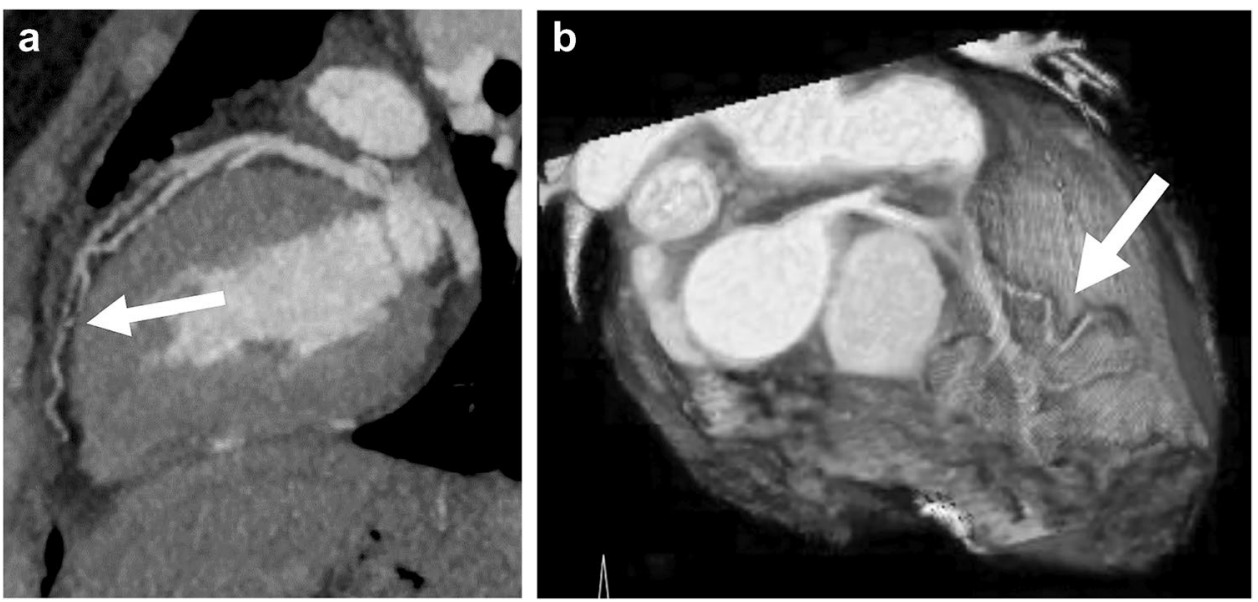
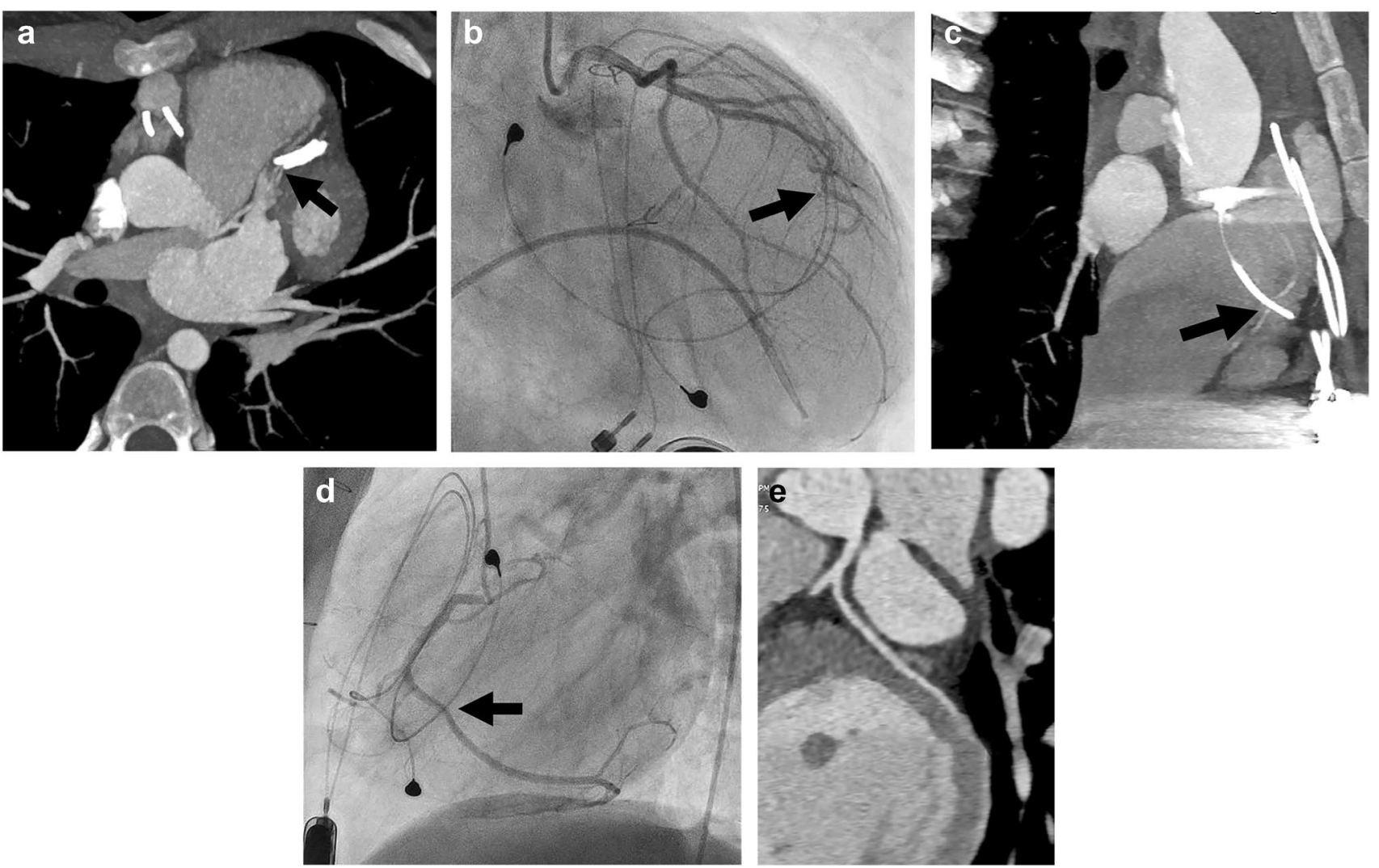

Fig. 8 Lead compression of the coronary arteries in a 13-year-old girl with epicardial pacer leads. a Axial oblique maximum-intensity projection (MIP) CT angiogram shows deviation and compression of the left anterior descending artery in the interventricular groove by an epicardial lead (arrow). b Catheter angiography in the right anterior oblique projection of a contrast injection of the left anterior descending (LAD) artery also demonstrates deviation and compression of the
LAD (arrow). c Sagittal oblique MIP CT angiogram of the right coronary artery shows compression by an epicardial lead (arrow). d This is also demonstrated on subsequent catheter angiography in the left lateral projection (arrow). e Curved planar reformatted CT angiogram of the left coronary artery and LAD artery following lead removal shows no significant sequela 


\section{Declarations}

\section{Conflicts of interest None}

\section{References}

1. Frommelt P, Lopez L, Dimas VV et al (2020) Recommendations for multimodality assessment of congenital coronary anomalies: a guide from the American Society of Echocardiography: developed in collaboration with the Society for Cardiovascular Angiography and Interventions, Japanese Society of Echocardiography, and Society for Cardiovascular Magnetic Resonance. J Am Soc Echocardiogr 33:259-294

2. Achenbach S, Marwan M, Schepis T et al (2009) High-pitch spiral acquisition: a new scan mode for coronary $\mathrm{CT}$ angiography. J Cardiovasc Comput Tomogr 3:117-121

3. Paul J-F, Rohnean A, Sigal-Cinqualbre A (2010) Multidetector CT for congenital heart patients: what a paediatric radiologist should know. Pediatr Radiol 40:869-875

4. Long CM, Long SS, Johnson PT et al (2015) Utility of low-dose high-pitch scanning for pediatric cardiac computed tomographic imaging. J Thorac Imaging 30:W36-40

5. Han BK, Rigsby CK, Leipsic J et al (2015) Computed tomography imaging in patients with congenital heart disease, part 2: technical recommendations. An expert consensus document of the Society of Cardiovascular Computed Tomography (SCCT): endorsed by the Society of [sic] Pediatric Radiology (SPR) and the North American Society of Cardiac Imaging (NASCI). J Cardiovasc Comput Tomogr 9:493-513

6. Han BK, Rigsby CK, Hlavacek A et al (2015) Computed tomography imaging in patients with congenital heart disease part I: rationale and utility. An expert consensus document of the Society of Cardiovascular Computed Tomography (SCCT): endorsed by the Society of [sic] Pediatric Radiology (SPR) and the North American Society of Cardiac Imaging (NASCI). J Cardiovasc Comput Tomogr 9:475-492

7. Zheng M, Zhao H, Xu J et al (2013) Image quality of ultra-lowdose dual-source CT angiography using high-pitch spiral acquisition and iterative reconstruction in young children with congenital heart disease. J Cardiovasc Comput Tomogr 7:376-382

8. Secinaro A, Curione D, Mortensen KH et al (2019) Dual-source computed tomography coronary artery imaging in children. Pediatr Radiol 49:1823-1839

9. Tomizawa N, Maeda E, Akahane M et al (2013) Coronary CT angiography using the second-generation 320-detector row CT: assessment of image quality and radiation dose in various heart rates compared with the first-generation scanner. Int J Cardiovasc Imaging 29:1613-1618

10. Huang M-P, Liang C-H, Zhao Z-J et al (2011) Evaluation of image quality and radiation dose at prospective ECG-triggered axial 256slice multi-detector CT in infants with congenital heart disease. Pediatr Radiol 41:858-866

11. Yao L-P, Zhang L, Li H-M et al (2017) Assessment of coronary artery by prospective ECG-triggered 256 multi-slice CT on children with congenital heart disease. Int J Cardiovasc Imaging 33:2021-2028

12. Le Roy J, Vernhet Kovacsik H, Zarqane H et al (2019) Submillisievert multiphasic coronary computed tomography angiography for pediatric patients with congenital heart diseases. Circ Cardiovasc Imaging 12:e008348

13. Gottumukkala RV, Kalra MK, Tabari A et al (2019) Advanced CT techniques for decreasing radiation dose, reducing sedation requirements, and optimizing image quality in children. Radiographics 39:709-726

14. Jadhav SP, Golriz F, Atweh LA et al (2015) CT angiography of neonates and infants: comparison of radiation dose and image quality of target mode prospectively ECG-gated 320-MDCT and ungated helical 64-MDCT. AJR Am J Roentgenol 204:W184-191

15. Ben Saad M, Rohnean A, Sigal-Cinqualbre A et al (2009) Evaluation of image quality and radiation dose of thoracic and coronary dual-source CT in 110 infants with congenital heart disease. Pediatr Radiol 39:668-676

16. Goo HW (2018) Combined prospectively electrocardiographyand respiratory-triggered sequential cardiac computed tomography in free-breathing children: success rate and image quality. Pediatr Radiol 48:923-931

17. Goo HW (2018) Identification of coronary artery anatomy on dual-source cardiac computed tomography before arterial switch operation in newborns and young infants: comparison with transthoracic echocardiography. Pediatr Radiol 48:176-185

18. Goo HW, Yang DH (2010) Coronary artery visibility in freebreathing young children with congenital heart disease on cardiac 64-slice CT: dual-source ECG-triggered sequential scan vs. single-source non-ECG-synchronized spiral scan. Pediatr Radiol 40:1670-1680

19. Jin KN, Park E-A, Shin C-I et al (2010) Retrospective versus prospective ECG-gated dual-source $\mathrm{CT}$ in pediatric patients with congenital heart diseases: comparison of image quality and radiation dose. Int J Cardiovasc Imaging 26:63-73

20. Kanie Y, Sato S, Tada A, Kanazawa S (2017) Image quality of coronary arteries on non-electrocardiography-gated high-pitch dual-source computed tomography in children with congenital heart disease. Pediatr Cardiol 38:1393-1399

21. Liu Y, Li J, Zhao H et al (2016) Image quality and radiation dose of dual-source CT cardiac angiography using prospective ECGtriggering technique in pediatric patients with congenital heart disease. J Cardiothorac Surg 11:47

22. Marukawa Y, Sato S, Tanaka T et al (2017) Evaluating low-kV dual-source CT angiography by high-pitch spiral acquisition and iterative reconstruction in pediatric congenital heart disease patients. Acta Med Okayama 71:407-412

23. Gao W, Zhong YM, Sun AM et al (2016) Diagnostic accuracy of sub-mSv prospective ECG-triggering cardiac CT in young infant with complex congenital heart disease. Int J Cardiovasc Imaging 32:991-998

24. Han BK, Lindberg J, Grant $K$ et al (2011) Accuracy and safety of high pitch computed tomography imaging in young children with complex congenital heart disease. Am J Cardiol 107:1541-1546

25. Han BK, Lindberg J, Overman D et al (2012) Safety and accuracy of dual-source coronary computed tomography angiography in the pediatric population. J Cardiovasc Comput Tomogr 6:252-259

26. Malone LJ, Olson A, Barker AJ et al (2020) Visualization of proximal coronary arteries on high-pitch electrocardiogramtriggered computed tomography in pediatric congenital heart disease: effects of heart rate and body surface area. Pediatr Radiol 50:1375-1380

27. Ghekiere O, Nchimi A, Djekic J et al (2016) Coronary computed tomography angiography: patient-related factors determining image quality using a second-generation 320 -slice CT scanner. Int J Cardiol 221:970-976

28. Bastarrika G, De Cecco CN, Arraiza M et al (2008) Dual-source $\mathrm{CT}$ for visualization of the coronary arteries in heart transplant patients with high heart rates. AJR Am J Roentgenol 191:448-454

29. Smettei OA, Sayed S, Al Habib M, A, et al (2018) Ultra-fast, low dose high-pitch (FLASH) versus prospectively-gated coronary computed tomography angiography: comparison of image quality and patient radiation exposure. J Saudi Heart Assoc 30:165-171 
30. Chelliah A, Kubacki T, Julien HM, Einstein AJ (2016) Pediatric coronary CTA using phenylephrine to lower heart rate. J Cardiovasc Comput Tomogr 10:339-340

31. Li T, Zhao S, Liu J et al (2017) Feasibility of high-pitch spiral dual-source $\mathrm{CT}$ angiography in children with complex congenital heart disease compared to retrospective-gated spiral acquisition. Clin Radiol 72:864-870

32. Jacobs ML, Mavroudis C (2010) Anomalies of the coronary arteries: nomenclature and classification. Cardiol Young 20:15-19

33. Cheezum MK, Liberthson RR, Shah NR et al (2017) Anomalous aortic origin of a coronary artery from the inappropriate sinus of Valsalva. J Am Coll Cardiol 69:1592-1608

34. Agarwal PP, Dennie C, Pena E et al (2017) Anomalous coronary arteries that need intervention: review of pre- and postoperative imaging appearances. Radiographics 37:740-757

35. Mery CM, De León LE, Molossi S et al (2018) Outcomes of surgical intervention for anomalous aortic origin of a coronary artery: a large contemporary prospective cohort study. J Thorac Cardiovasc Surg 155:305-319.e4

36. Vastel-Amzallag C, Le Bret E, Paul J-F et al (2011) Diagnostic accuracy of dual-source multislice computed tomographic analysis for the preoperative detection of coronary artery anomalies in 100 patients with tetralogy of Fallot. J Thorac Cardiovasc Surg 142:120-126

37. Yu F-F, Lu B, Gao Y et al (2013) Congenital anomalies of coronary arteries in complex congenital heart disease: diagnosis and analysis with dual-source CT. J Cardiovasc Comput Tomogr 7:383-390

38. Pandey NN, Sinha M, Sharma A et al (2019) Anomalies of coronary artery origin: evaluation on multidetector CT angiography. Clin Imaging 57:87-98

39. McCrindle BW, Rowley AH, Newburger JW et al (2017) Diagnosis, treatment, and long-term management of Kawasaki disease: a scientific statement for health professionals from the American Heart Association. Circulation 135:e927-e999

40. Newburger JW, Takahashi M, Beiser AS et al (1991) A single intravenous infusion of gamma globulin as compared with four infusions in the treatment of acute Kawasaki syndrome. N Engl J Med 324:1633-1639
41. Newburger JW, Takahashi M, Burns JC et al (1986) The treatment of Kawasaki syndrome with intravenous gamma globulin. N Engl J Med 315:341-347

42. Sperotto F, Friedman KG, Son MBF et al (2021) Cardiac manifestations in SARS-CoV-2-associated multisystem inflammatory syndrome in children: a comprehensive review and proposed clinical approach. Eur J Pediatr 180:307-322

43. Warnes CA, Williams RG, Bashore TM et al (2008) ACC/AHA 2008 guidelines for the management of adults with congenital heart disease: a report of the American College of Cardiology/ American Heart Association task force on practice guidelines (writing committee to develop guidelines on the management of adults with congenital heart disease). Developed in collaboration with the American Society of Echocardiography, Heart Rhythm Society, International Society for Adult Congenital Heart Disease, Society for Cardiovascular Angiography and Interventions, and Society of Thoracic Surgeons. J Am Coll Cardiol 52:e143-e263

44. Marano R, Rovere G, Savino G et al (2020) CCTA in the diagnosis of coronary artery disease. Radiol Med 125:1102-1113

45. Rohnean A, Houyel L, Sigal-Cinqualbre A et al (2011) Heart transplant patient outcomes: 5 -year mean follow-up by coronary computed tomography angiography. Transplantation 91:583

46. Carreras EM, Duncan WJ, Djurdjev O, Campbell AIM (2015) Cardiac strangulation following epicardial pacemaker implantation: a rare pediatric complication. J Thorac Cardiovasc Surg 149:522-527

47. Mah DY, Prakash A, Porras D et al (2018) Coronary artery compression from epicardial leads: more common than we think. Heart Rhythm 15:1439-1447

Publisher's Note Springer Nature remains neutral with regard to jurisdictional claims in published maps and institutional affiliations. 